\title{
Total Least Squares Registration of 3D Surfaces
}

\author{
Umut Aydar, M. Orhan Altan*
}

Istanbul Technical University, Faculty of Civil Engineering, Department of Geomatics Engineering, ISTANBUL-TR

Corresponding author.* Tel: +90212 2853414

Received 01 Apr 2015

E-mail: oaltan@itu.edu.tr

Accepted 01 Jun 2015

\begin{abstract}
Co-registration of point clouds of partially scanned objects is the first step of the 3D modeling workflow. The aim of co-registration is to merge the overlapping point clouds by estimating the spatial transformation parameters. In computer vision and photogrammetry domain one of the most popular methods is the ICP (Iterative Closest Point) algorithm and its variants. There exist the 3D Least Squares (LS) matching methods as well (Gruen and Akca, 2005). The co-registration methods commonly use the least squares (LS) estimation method in which the unknown transformation parameters of the (floating) search surface is functionally related to the observation of the (fixed) template surface. Here, the stochastic properties of the search surfaces are usually omitted. This omission is expected to be minor and does not disturb the solution vector significantly. However, the a posteriori covariance matrix will be affected by the neglected uncertainty of the function values of the search surface. . This causes deterioration in the realistic precision estimates. In order to overcome this limitation, we propose a method where the stochastic properties of both the observations and the parameters are considered under an errors-in-variables (EIV) model. The experiments have been carried out using diverse laser scanning data sets and the results of EIV with the ICP and the conventional LS matching methods have been compared.
\end{abstract}

Keywords: Laser scanning, Point Cloud, Registration, Matching, Total Least Squares

\section{Introduction}

3D object modeling plays an important role for many applications from reverse engineering to creating the real-world models for virtual reality, architecture or deformation analysis. In the last decade, laser scanners had an utmost importance for 3D object modeling due to their ability of providing reliable 3D data very fast and directly. Since the range scanners are lineof-sight instruments, in many cases an object has to be scanned from different standpoints to be able to cover the whole object. As a result, separate point clouds, which are in their own local co-ordinate systems uniquely, are obtained. In order to form a 3D model, these point clouds have to be merged in one coordinate system. This process is called alignment or registration. Various methods were proposed and the studies in this area are still in progress especially in computer vision discipline including the most popular Iterative Closest Point (ICP) algorithm and its variants. Since the introduction of ICP by Chen and
Medioni, (1991) and Besl and McKay, (1992), many variants have been introduced on the basic ICP concept. A detailed review of the ICP variants can be found at Akca, (2010) and Rusinkiewicz, (2001). Despite the popularity of the ICP, there are some disadvantageous aspects of it in terms of accuracy assesment of transformation parameters. ICP based algorithms generally uses closed-form solutions for the estimation of transformation parameters. The closed-form solutions cannot fully consider the statistical accuracy assesment of the estimated parameters. One another powerfull and adaptive method for the registration problem is the $3 \mathrm{D}$ least squares surface matching proposed by Gruen and Akca, in (2005). The method is the extension and adaptation of mathematical model of Least Squares 2D image matching for the 3D surface matching problem. The transformation parameters of the search surfaces are estimated with respect to a template surface. The solution is achieved when the sum of the squares of the 3D spatial (Euclidean) distances between the 
surfaces are minimized. The parameter estimation is achieved using the Generalized Gauss-Markov model. Akca, (2010). At this model, the points on the template surface are considered as observations, contaminated by random errors, while the search surface points are assumed as error-free.

$y+e \_y=t+R * x$

With the assumptions

e_y N $\left(0, \sigma \_0 \wedge 2 \llbracket P \_y \rrbracket \rrbracket^{\wedge}(-1)\right)$

where $\mathrm{y}$ is the template point, $\mathrm{x}$ is the search point, ey is the true error vector for template points, $\mathrm{t}$ is the translation vector, $\mathrm{R}$ is the rotation matrix, and $\mathrm{P}$ is the weight matrix. Here, and also in the ICP methods, the stochastic properties of the search surfaces are usually omitted. This omission is expected to be minor and does not disturb the solution vector significantly. However, the a posteriori covariance matrix will be affected by the neglected uncertainty of the function values of $x$. This causes deterioration in the realistic precision estimates. More details on this issue can be found in Gruen, (1985), Maas, (2002), Gruen and Akca, (2005), Kraus et al., (2006), and Akca, (2010). These algorithms consider the noise as coming from one measurement only, but in fact both surface measurements are corrupted by noise. To be able to overcome this undesirable situation and obtain more realistic precision estimation values, another approach which takes the stochastic properties of the elements of design matrix into consideration should be applied. The problem can be solved by using a model which is called in the literature as Errors-in-Variables model or the total least squares (TLS). Markovsky and Huffel, (2007) outlines the different solution methods and application areas of EIV model very detailed. Ramos and Verriest, (1997) proposed to use the total least squares approach for the registration of m-D data. In their study, they use a mixed solution which is the combination of Least squares and Total Least squares methods for the registration of 2D medical images. However, they do not give any information about the precision of the transformation parameters. Aky1lmaz, (2007) uses Total Least Squares method for coordinate transformation in Geodetic applications. Since the author uses a closed-form solution method in this study, there is not any information about precision of estimated parameters as well. A mathematical model is given by Neitzel, (2007) where an iterative Gauss-Helmert type of adjustment model with the linearized condition equations is adopted. However, in this method the size of the normal equations to be solved increases dramatically depending on the number of conjugate points, since each point introduces three more Lagrange multipliers into the normal equations. Thus, the larger the number of conjugate points, the greater the normal equations to be solved.

For an optimal solution of the so-called EIV problem, we propose a modified iterative Gauss-Helmert type of adjustment model. In this model, the rotation matrix $\mathrm{R}$ is represented in terms of unit quaternions $q=\left[\begin{array}{llll}q 0 & \text { q1 } & \text { q2 } & \text { q3}\end{array}\right]$. Moreover, the dimension of the normal equations to be solved is dramatically reduced to the number of unknown transformation parameters which is six for the rigid-body transformation problem. The mathematical model has been implemented in MATLAB. This study mainly aims at comparing the proposed model with LS matching method in terms of the precision of estimated parameters by using diverse laser scanning data sets.

\section{Errors-In-Variables Model}

The aim of co-registration process is to transform search surface with respect to the template surface by establishing the correspondences between two overlapping data sets. Assuming the existence of two overlapping 3D data sets $\mathrm{Qi}$ and $\mathrm{Pj}(\mathrm{i}=1, \ldots, \mathrm{N}$ and $\mathrm{j}=1, \ldots, \mathrm{M})$, we can find a corresponding point in $\mathrm{Pj}$ for each point in $\mathrm{Qi}$ by using different error metrics, which $\mathrm{Qi}$ and $\mathrm{Pj}$ are the template and search surfaces respectively. Once the appropriate correspondences were established between two point data sets the basic procedure is to estimate the transformation parameters using the correspondences. The geometric relationship is established by a six parameters 3D rigid-body transformation (3). 
$\left[\begin{array}{c}X_{i} \\ Y_{i} \\ Z_{i}\end{array}\right]=\left[\begin{array}{c}T x \\ T y \\ T z\end{array}\right]+R *\left[\begin{array}{c}x_{j} \\ y_{j} \\ z_{j}\end{array}\right]$

In the classical Gauss-Markov model, Eq. (1) represents the observation equation which assumes the template surface elements are observations contaminated by random the errors. In fact, the search surface elements are also erroneous and a true error vector should be added to these elements as well. The observation equations in EIV model are formed as

$y+e \_y=t+R *\left(x+e \_x\right)$.

If we apply this model to $3 \mathrm{D}$ rigid-body transformation, the mathematical model is established as;

$l+v_{x}=\left(A+v_{A}\right) * \beta$

where $\boldsymbol{v}_{x}$ is the $n \times 1$ residual vector of observations and $\boldsymbol{v}_{A}$ is an $n \times m$ error matrix of the corresponding elements of design matrix. The elements of both $\boldsymbol{v}_{x}$ and $\boldsymbol{v}_{A}$ are independent and conforming the normal distributed with zero mean. Once a minimisation of $\left[\tilde{v}_{A} ; \tilde{v}_{x}\right]$ is found, then any $\beta$ satisfying

$\left(\mathrm{A}+\tilde{v}_{A}\right) \cdot \beta=l+\tilde{v}_{x}$ is the solution of the problem by Total Least Squares.

\section{Proposed Modified Gauss-Helmert Model}

The generalized total least squares solution of the 3D-similarity transformation by introducing the quaternions as the representation of the rotation matrix*scale factor $(S=s R)$ based on iteratively linearized Gauss-Helmert model has been presented by Akyilmaz, (2010, 2011). However, this model requires the solution of a normal matrix which includes the corresponding terms for transformation parameters as well as the Lagrange multipliers, thus yielding a larger size of system of equations to be solved at each iteration with the increase of the identical points of the transformation problem. Following the idea in Akyilmaz, (2010 and 2011), Kanatani and Niitsuma, (2012) has developed a new computational scheme for 3D-similarity transformation which they call Modified Iterative Gauss-
Helmert model by reducing the so-called Lagrange multipliers and hence the size of the normal matrix is dramatically reduced. In other words, the unknowns to be solved at each iteration are equal to seven, i.e. the number of transformation parameters. This kind of a reduction provides advantage, especially in terms of computational aspects. We refer to Kanatani and Niitsuma, (2012) for details of the mathematical model. Modified Gauss-Helmert model in Kanatani and Niitsuma, (2012) is a seven parameters similarity transformation. Therefore, in our study, we modified the model by eliminating the scale factor in order to apply 6 parameters rigid-body transformation. For this purpose we normalise the quaternion by using the $\mathrm{q}_{0}{ }^{2}+\mathrm{q}_{1}{ }^{2}+\mathrm{q}_{2}{ }^{2}+\mathrm{q}_{3}{ }^{2}=1$ equality. Then the rotation matrix defined by quaternions is obtained as;

$\mathrm{S}=$

$\left[\begin{array}{ccc}2 \mathrm{q}_{2}{ }^{2}-2 \mathrm{q}_{3}{ }^{2}+1 & 2 q_{1} q_{2}-2 q_{3} N & 2 q_{2} N+2 q_{1} q_{3} \\ 2 q_{3} N+2 q_{1} q_{2} & -2 q_{1}^{2}-2 \mathrm{q}_{3}^{2}+1 & 2 q_{1} N \\ 2 q_{1} q_{3}-2 q_{2} N & 2 q_{1} N+2 q_{2} q_{3} & 2 \mathrm{q}_{2}^{2}+1\end{array}\right]$

(6)

$N=$

$\sqrt{\left(-q_{1}^{2}-q_{2}^{2}-q_{3}^{2}+1\right)}$

(7)

In so-called model, let $a_{i}$ and $b_{i}$ are the corresponding pairs $(\mathrm{i}=1, \ldots, \mathrm{M}) ; \mathrm{Q}_{\mathrm{xx}}\left[\mathrm{a}_{\mathrm{i}}\right]$ and $\mathrm{Q}_{\mathrm{xx}}\left[\mathrm{b}_{\mathrm{i}}\right]$ are normalized covariance matrices; $\bar{a}_{i}$ and $\bar{b}_{i}$ are the true positions of $\mathrm{a}_{\mathrm{i}}$ and $\mathrm{b}_{\mathrm{i}}$ respectively. The optimal estimation of the similarity transformation parameters $\mathrm{R}$ (rotation), $\mathrm{T}$ (translation) and $\mathrm{s}$ (scale factor) in the sense of Maximum Likelihood is to minimize the Mahalanobis distance given as follows.

$J=\frac{1}{2} \sum_{i=1}^{M}\left(a_{i}-\bar{a}_{\imath}\right)^{T} Q_{x x}{ }^{-1}\left[a_{i}\right]\left(a_{i}-\right.$

$\left.\overline{a_{l}}\right)+\ldots$.

$\ldots+\frac{1}{2} \sum_{i=1}^{M}\left(b_{i}-\bar{b}_{\imath}\right)^{T} Q_{x x}{ }^{-1}\left[b_{i}\right]\left(b_{i}-\bar{b}_{\imath}\right)$

and

$\bar{a}_{i}=S \bar{b}_{i}+T$ 
Where $\mathrm{S}$ is the rotation matrix given in (6)

Since the model is non-linear, it is linearized by the Taylor Series expansion. Finally, the total error vector is defined as

$e_{i}=a_{i}-\mathrm{S} b_{i}-\mathrm{T}$

With the weight matrix;

$W_{i}=\left(S Q_{x x}\left[a_{i}\right] S^{\prime}+Q_{x x}\left[b_{i}\right]\right)^{-1}$

After modifications, Eq. 7 can be expressed in the following form:

$J=\frac{1}{2} \sum_{1}^{M}\left(e_{i}{ }^{\prime} W_{i} e_{i}\right)$

Differentiating (6) with respect to qi, $i=1,2$, 3

$\frac{\partial S}{\partial q_{i}}=2 Q_{i}$

We define a $3 \times 3 \mathrm{U}_{\mathrm{i}}$ matrix as follows

$U_{i}=\left[\begin{array}{lll}Q_{1} b_{i} & Q_{2} b_{i} & Q_{3} b_{i}\end{array}\right]$

After these definitions, parameters are estimated by the solution of following 6-D linear equation.

$$
\begin{aligned}
& \left(\begin{array}{cc}
\sum_{1}^{M} U_{i}{ }^{T} W_{i} U_{i} & \sum_{1}^{M} U_{i}{ }^{T} W_{i} \\
\sum_{1}^{M} W_{i} U_{i} & \sum_{1}^{M} W_{i}
\end{array}\right)\left(\begin{array}{c}
\Delta q \\
\Delta T
\end{array}\right)= \\
& \left(\begin{array}{c}
\sum_{1}^{M} U_{i}{ }^{T} W_{i} e_{i} \\
\sum_{1}^{M} W_{i} e_{i}
\end{array}\right)
\end{aligned}
$$

Since so-called model is non-linear, initial approximations of $\mathrm{q}$ and $\mathrm{T}$ are updated and iteration is repeated until it converges.

\section{Stochastic Model}

The studies on calibration of laser scanners (Lichti 2007) and laser scanner accuracy show that there are many factors affecting on the individual point precision of terrestrial laser scanners like instrument's range and angular accuracy, geometric factors (e.g. edge effect and incidence angle), atmospheric conditions (humidity, temperature etc.) and radiometric effects (surface reflectivity etc.). In our implementation, we define a covariance matrix for each individual point by using the range and angular accuracy of the scanner and incidence angle. Since the Cartesian coordinates are derived quantities, we can transform back them into the spherical coordinates ( $\rho=$ range, $\theta=$ horizontal angle and $\varphi=$ vertical angle) basically by using the equations

$$
\begin{aligned}
& \rho_{i}=\sqrt{x_{i}^{2}+y_{i}^{2}+z_{i}^{2}} \\
& \theta_{i}=\arctan \left(\frac{y_{i}}{x_{i}}\right) \\
& \varphi_{i}=\arctan \left(\frac{z_{i}}{\sqrt{x_{i}^{2}+y^{2}}}\right) \\
& r_{i}=\left[\rho_{i} \theta_{i} \varphi_{i}\right]
\end{aligned}
$$

Thus we can write the Cartesian coordinates of a point

in terms of spherical coorinates as (17);

$$
P i \quad\left\{\begin{array}{c}
P x=\rho i * \cos (\theta \mathrm{i}) * \cos (\varphi \mathrm{i}) \\
P y=\rho i * \cos (\varphi \mathrm{i}) * \sin (\theta \mathrm{i}) \\
P z=\rho i * \sin (\varphi \mathrm{i})
\end{array}\right\}
$$

The $3 \times 3$ covariance matrix of point is obtained from propagating the precision of the original spherical observables, which are typically provided by the manufacturer (Grant). The variances of these spherical observables are $\sigma_{P_{i}} \sigma_{\theta_{i}} \sigma_{i}$ respectively. Precision values of spherical coordinates provided by the vendor are (18);

$$
\sum r=\left|\begin{array}{lll}
\sigma_{P_{i}}{ }^{2} & & \\
& \sigma_{\theta_{i}}{ }^{2} &
\end{array}\right|
$$

The second factor effecting on the point precision is incidence angle $\alpha$, which is defined as the angle between the laser beam and local surface normal on related point. Simply it could be explained as the orientation of the local surface with respect to the scanner position. (Soudarissanane et al., 2011) explain that the incidence angle and range accuracy are inversely proportional. So, 
we update our range measurement accuracy by dividing the range variance by cosine of the incidence angle. By replacing the first element in (19) with

$$
\left(\frac{\sigma_{P_{i}}}{\cos (\alpha)}\right)^{2}
$$

we obtain a new precision variance matrix for point $r_{i}, \sum r_{n e w}$. If we apply the error propagation rule, we obtain the covariance matrix for point $P_{i}$ from (20);

$$
Q_{x x}=F \sum r_{\text {new }} \times F^{T}
$$

Where $F$ is the Jacobian matrix of $P_{i}$ with respect to $\rho_{i} \theta_{i}$ and $\varphi_{i}$ respectively (21).

$$
F=\left[\begin{array}{ccc}
\cos (\varphi) \cos (\theta) & -\rho \cos (\varphi) \sin (\theta) & -\rho \cos (\theta) \sin (\varphi) \\
\cos (\varphi) \sin (\theta) & \rho \cos (\varphi) \cos (\theta) & -\rho \sin (\varphi) \sin (\theta) \\
\sin (\varphi) & 0 & \rho \cos (\varphi)
\end{array}\right]
$$

\section{Correspondence Search}

Correspondence search is the most critical part of all registration algorithms. The succes of a registration method depends on how correct correspondences were established between two data sets. False matches cause to uncorrect results. In order to prevent false matches, different type of constraints can be introduced. In our implementation, the correspondence search is guided by using two well-known error metrics. The first one is the point-to-point search which was introduced by Besl and McKay (1992) in their original ICP paper. According to this method, each available point in template surface is matched with the closest point in search surface. Then, the sum of the squared distances between the points in each correspondence pair is minimized. This procedure is very complex in terms of computational aspects and takes the most of the computation time. The procedure has been accelerated by using a kd-tree searcher in our implementation. The second error metric is the point-to-plane algorithm which was introduced by Chen and Medioni (1991). In point-to-plane error metric, the sum of the squared distances between each point in template data and the tangent plane at its corresponding destination point in search data is minimized. Due to the large search area and heavy mathematical computations like plane parameters, surface normal and Euclidian distance calculation, point-to-plane error metric is much slower than point-to-point version. On the other hand, the researchers have observed significantly better convergence rates with point-to-plane (Rusinkiewicz, 2001). One solution for accelerating the point-to-plane version is to limit the search area of the candidgte (point at the search surface. Based on the advantageous parts of these two versions, both of them were used together in this study in order to benefit from the advantageous parts of them. The point-to-plane search was accelerated significantly by using a kd-tree nearest neighbor searcher. The coarse match point is found by the point-to-poin 3.11 search; consequently the procedure is followed by the point-to-plane search where the fine matching point is found. The fine matching point is searched inside the 6 neighboring triangles which are fictitiously formed around the coarse matching point. The fine matching point should lie inside of one of those six triangles, and should have the minimum spatial distance to the corresponding template point. Any point satisfying the both conditions are labeled as the fine matching point.

\section{Experimental Results}

All experiments were carried out by using a self-developed programme in MATLAB Computing Language environment.

For the simplicity we name this programme as TLS3D throughout this section. We also coded the classical Least Squares in MATLAB as well which we call LS shortly. Our purpose is to investigate the results of proposed MGH model for the registration problem and make comparisons between the results obtained by LS.

We conducted several tests by using diverse laser scanning data acquired by different 
devices which have alternate working principles, accuracy specifications and noise characteristics. Besides, we used some synthetic data in order to control the results of programmes.

In all experiments, initial approximations were provided beforehand by the help of external software (Leica cyclone, LS3D [Akca]) since we did not create a Graphical User Interface (GUI) for selecting some control points on both data. The developed software can handle the rotational differences about three axes up to $20^{\circ}$, but it is not that much capable about the translation vector.

The experiments in TLS3D can be classified into three in terms of the introduced initial covariance matrix. The first group of tests are conducted by using the same covariance matrices and isotropic noise for all sets. The second tests are carried out by using different covariance matrices for template and search surfaces with isotropic noise and the final group of tests are with different covariance matrices with anisotropic noise as explained

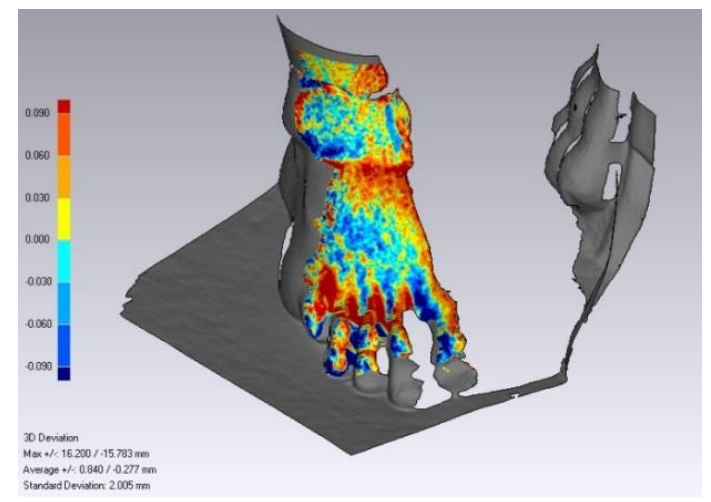

(a) in stochastic model chapter.

\section{Weary Heracles Statue}

The first experiment is the registration of two partial surfaces which is a part of "Weary Heracles" statue. The data has been scanned by Breuckmann optoTOP-HE coded structured light system. Original data set comprises approximately 250000 points individually with $0.5 \mathrm{~mm}$ point spacing. In this test we resampled the data set and decreased the number of points since our implementation produces the results very slowly with this kind of an intense data. Resampled data consist of approximately 50000 points with $1 \mathrm{~mm}$ point spacing. The matching process was carried out by using both LS and TLS3D with homogeneous and isotropic noise conditions. The obtained results are given in Table (1). Figure 1 shows the 3D comparison of two methods by using the 3D compare module of Geomagic Studio (Raindrop Geomagic, Inc.) software. Patterns of the residuals show similarity for both methods.

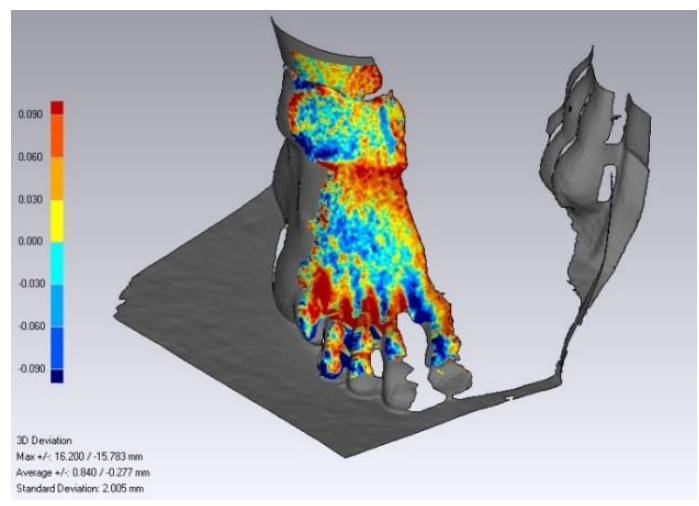

(b)

Figure 1. (a) is the template and (b) is the search surface. 
Table 1. Numerical results of 'Weary Heracles' data

\begin{tabular}{llccccccc}
\hline Model & $\begin{array}{l}\text { No. of } \\
\text { Matched } \\
\text { Points }\end{array}$ & $\begin{array}{c}\sigma_{0} \\
(\mathrm{~mm})\end{array}$ & $\begin{array}{c}\sigma_{T_{x}} \\
(\mathrm{~mm})\end{array}$ & $\begin{array}{c}\sigma_{T_{y}} \\
(\mathrm{~mm})\end{array}$ & $\begin{array}{l}\sigma_{T_{z}} \\
(\mathrm{~mm})\end{array}$ & $\begin{array}{c}\sigma_{\omega} \\
(\mathrm{grad})\end{array}$ & $\begin{array}{c}\sigma_{\varphi} \\
\text { (grad) }\end{array}$ & $\begin{array}{c}\sigma_{\kappa} \\
\text { (grad) }\end{array}$ \\
\hline TLS_isotropic & 36941 & 0.0292 & 0.00034 & 0.00040 & 0.00028 & 0.000006 & 0.000006 & 0.000004 \\
LS & 36856 & 0.0408 & 0.00033 & 0.00040 & 0.00028 & 0.000006 & 0.000006 & 0.000004 \\
\hline
\end{tabular}

\section{Surface patch}

The second experiment is the matching of two surface patches (Fig. 3). The data is acquired by an IMAGER 5003 terrestrial laser scanner (ZoellerFröchlich). The average point spacing

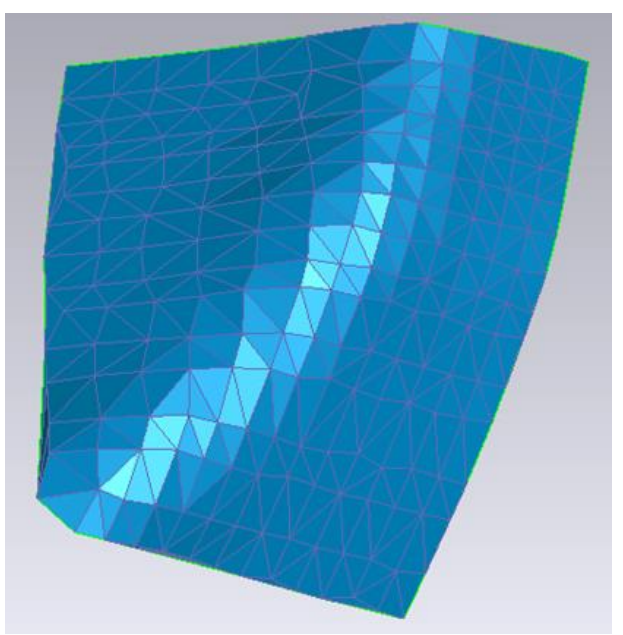

(a) is $1 \mathrm{~cm}$. Obtained numerical results for two different registrations are given in Table (2). In this experiment also, although the theoretical precisions changes slightly, differences are minor and insufficient for a decision making for the comparison purpose of two methods.

Figure 2. (a) is the template and (b) is the search surface.

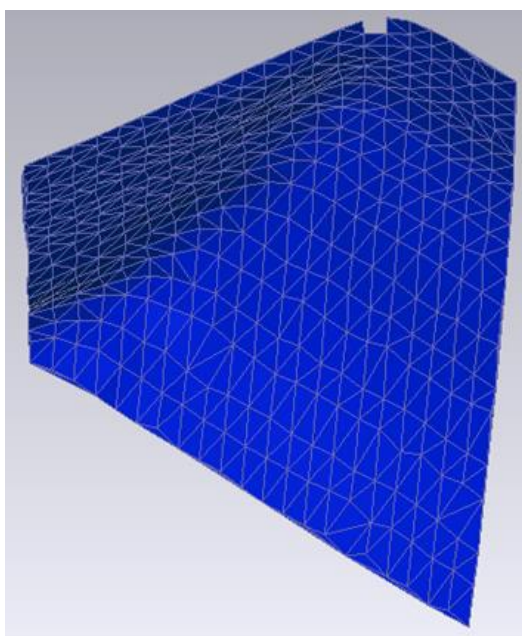

(b)

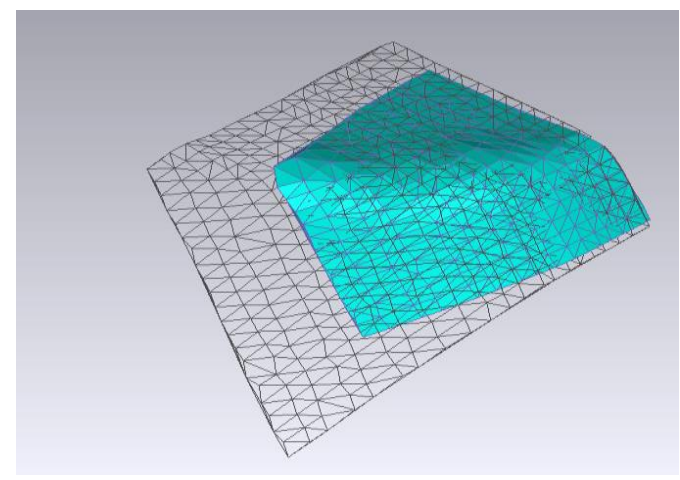

(a)

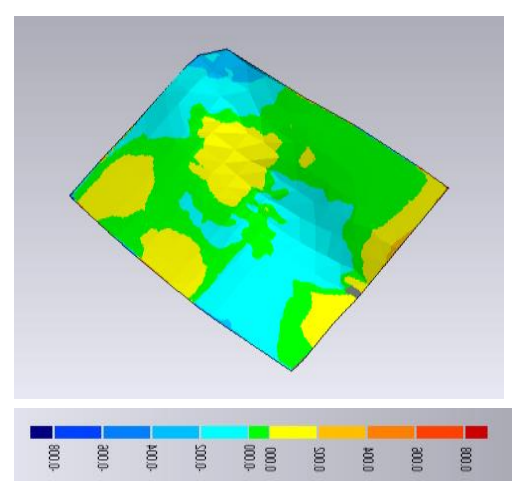

(b)

Figure 3. (a) is the registration with TLS ans (b) shows the residuals. 
Table 2. Numerical results of 'surface patch' data

\begin{tabular}{|c|c|c|c|c|c|c|c|c|}
\hline $\begin{array}{l}\text { Model } \\
\sigma_{\varphi}\end{array}$ & \multicolumn{2}{|l|}{ No. of } & \multicolumn{2}{|l|}{$\sigma_{0}$} & $\sigma_{T_{x}}$ & $\sigma_{T_{y}}$ & $\sigma_{T_{z}}$ & \multirow{2}{*}{$\begin{array}{r}\sigma_{\omega} \\
(\operatorname{deg})\end{array}$} \\
\hline $\begin{array}{l}\sigma_{\varphi} \\
(\operatorname{deg})\end{array}$ & $\begin{array}{c}\quad \sigma_{\kappa} \\
\text { Points }\end{array}$ & & $(\mathrm{mm})$ & $(\mathrm{mm})$ & $(\mathrm{mm})$ & $(\mathrm{mm})$ & (deg) & \\
\hline $\begin{array}{l}\text { TLS_is } \\
0.0001\end{array}$ & $\begin{array}{l}\text { tropic } \\
10\end{array}$ & 458 & 0.024763 & 0.002887 & 0.002883 & 0.004862 & 0.000244 & 0.000244 \\
\hline $\begin{array}{l}\text { LS } \\
0.0001\end{array}$ & & 544 & 0.035112 & 0.002510 & 0.002511 & 0.004239 & 0.000211 & 0.000210 \\
\hline
\end{tabular}

\section{Pyramid}

The third experiment is the registration of two scans of a small pyramid shape object. The half of the pyramid was scanned by Leica C10 scanner from close range. The average point spacing is $4 \mathrm{~mm}$. This data set can be considered as difficult for point to plane search because the object is consist of three planar surfaces which means it does not contain sufficient surface characteristics in terms of the orientation of the surface normal.
Moreover, the worst initial approximations $\left(\sim 18^{\circ}\right.$ rotation about $\mathrm{z}$ axis) among the all tests were introduced for this data. In this experiment, we used a-priori covariance matrices for both data sets calculated as explained in stochastic model section. Registration process is successful. The results of the test are given in Table (3). Results of the test show that we obtain more realistic a-posteriori precision values for estimated parameters.

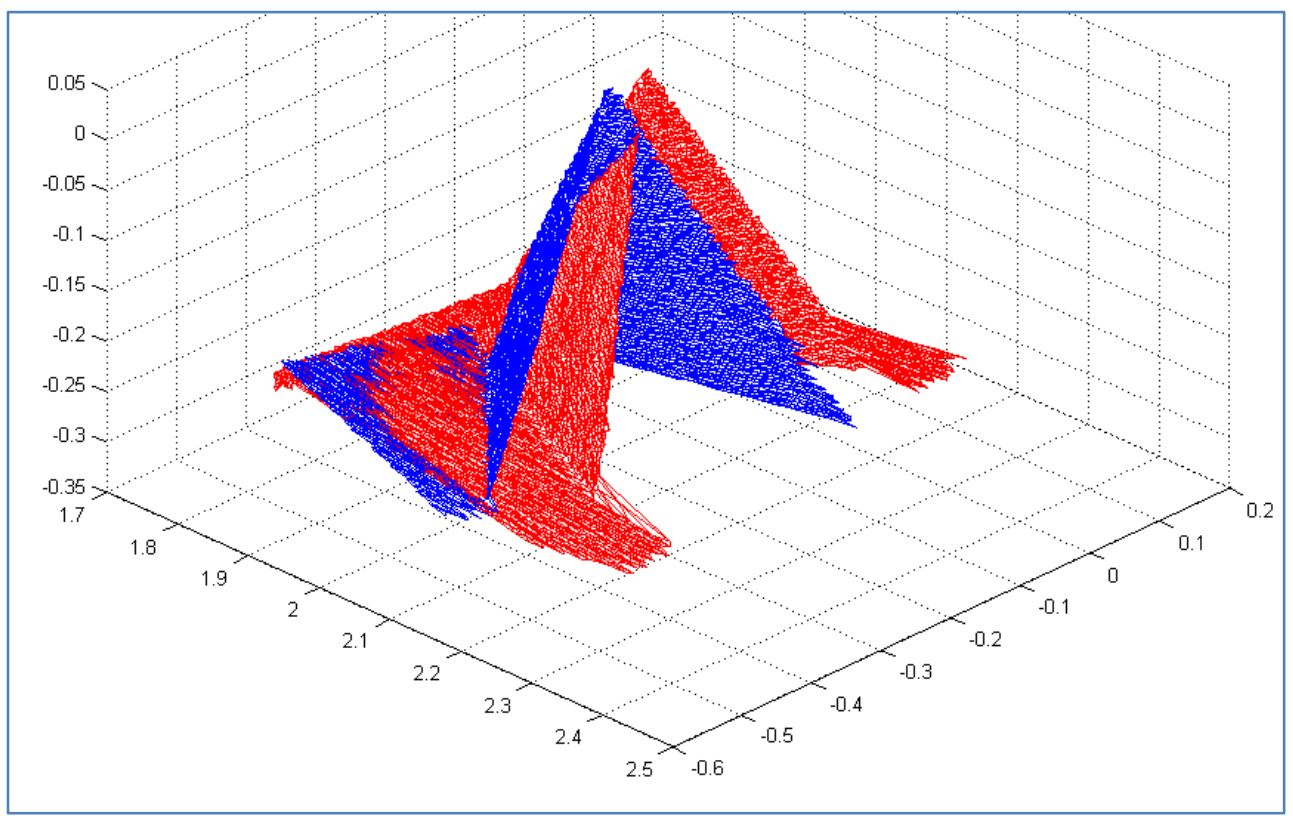

Figure 4. Template and search data. 


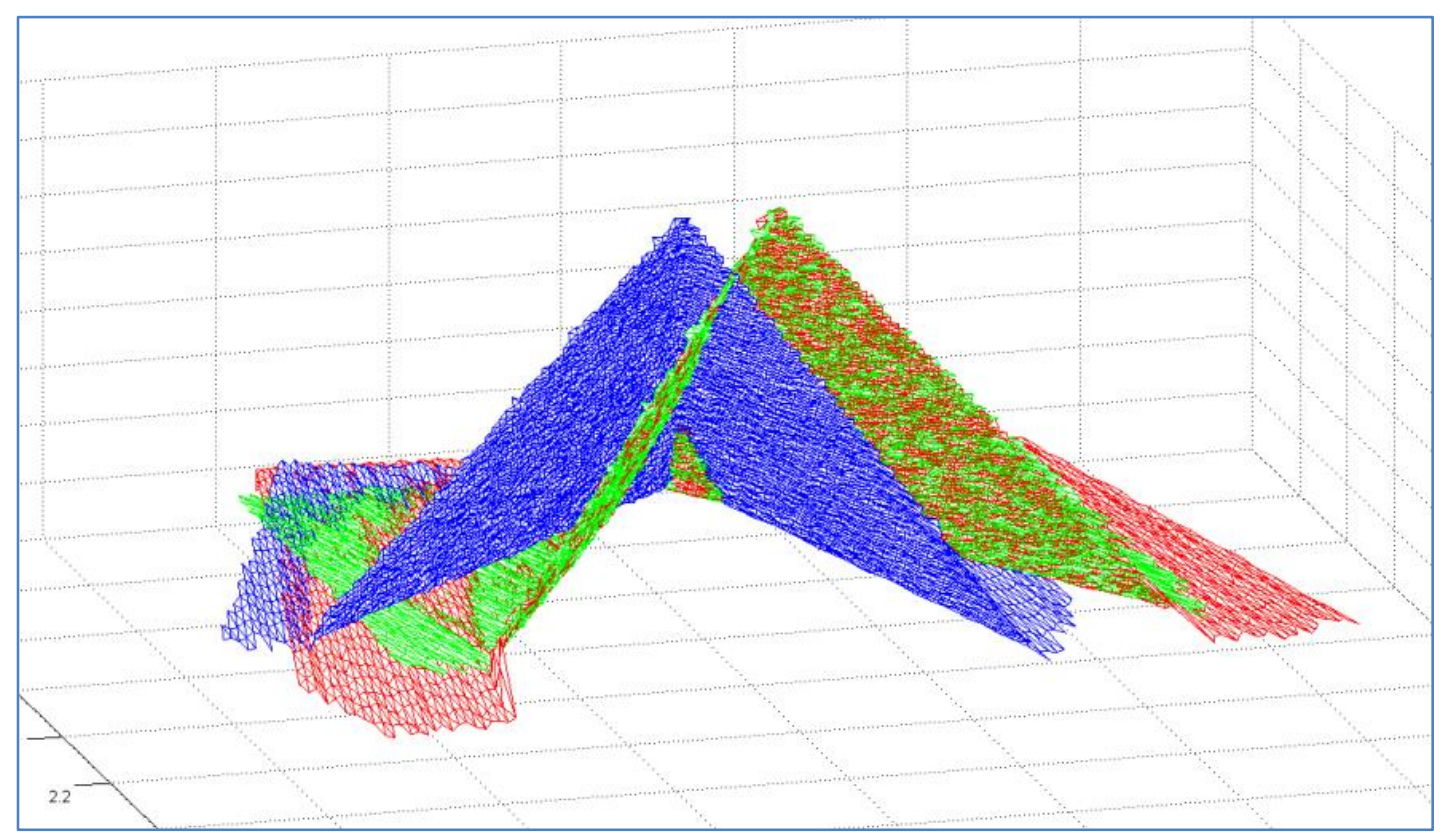

Figure 5. Registration result.

Table 3. Numerical results of 'pyramid' data

\begin{tabular}{lllllllll}
\hline \hline $\begin{array}{l}\text { Mod } \\
\text { el }\end{array}$ & $\begin{array}{l}\text { No. of } \\
\text { Matche } \\
\text { d }\end{array}$ & $\begin{array}{l}\sigma_{0} \\
(\mathrm{~m})\end{array}$ & $\begin{array}{c}\sigma_{T_{x}} \\
(\mathrm{~m})\end{array}$ & $\begin{array}{l}\sigma_{T_{y}} \\
(\mathrm{~m})\end{array}$ & $\begin{array}{c}\sigma_{T_{z}} \\
(\mathrm{~m})\end{array}$ & $\begin{array}{c}\sigma_{\omega} \\
(\mathrm{m})\end{array}$ & $\begin{array}{c}\sigma_{\varphi} \\
(\mathrm{m})\end{array}$ & $\begin{array}{c}\sigma_{\kappa} \\
(\mathrm{m})\end{array}$ \\
& Points & & & & & & & \\
TLS & 4960 & $\begin{array}{l}0.14443 \\
7\end{array}$ & $\begin{array}{l}0.00004 \\
5\end{array}$ & $\begin{array}{l}0.00024 \\
1\end{array}$ & $\begin{array}{l}0.00024 \\
7\end{array}$ & $\begin{array}{l}0.00008 \\
6\end{array}$ & $\begin{array}{l}0.00012 \\
4\end{array}$ & $\begin{array}{l}0.00011 \\
9\end{array}$ \\
\hline
\end{tabular}

\section{Wave data}

The fourth experiment is the registration process by using synthetic data. The data is called 'wave data' and is highly used by many researchers in order to test their registration algorithms (Salvi et. al. 2007, Rusinkiewitz 2001, Grant 2012). The data was downloaded from the MATLAB registration toolbox by Salvi et al. (2007). Transformation was simulated by applying rotation and transformation to data. Then we created anisotropic independent Gaussian noise by using the covariance definition given in stochastic model chapter. Obtained noises were added to both template and search surfaces. The registration process was carried out for three times. In first run, we used TLS3D without introducing the pre-defined covariance matrices as input. In second run, we use LS and for the third run we use again TLS3D by introducing the covariance matrices as input. Registration is successful for all runs. Numerical results of the tests are given in Table (4). Results are quite similar and consistent with the other experiments. Results do not change in significant level for LS and TLS3D without using a-priori covariance definition. But at the last run, the effects of the introduced anisotropic and non-homogeneous covariance matrices are observed. The obtained a-posteriori covariance matrices are smaller than the other two runs. 


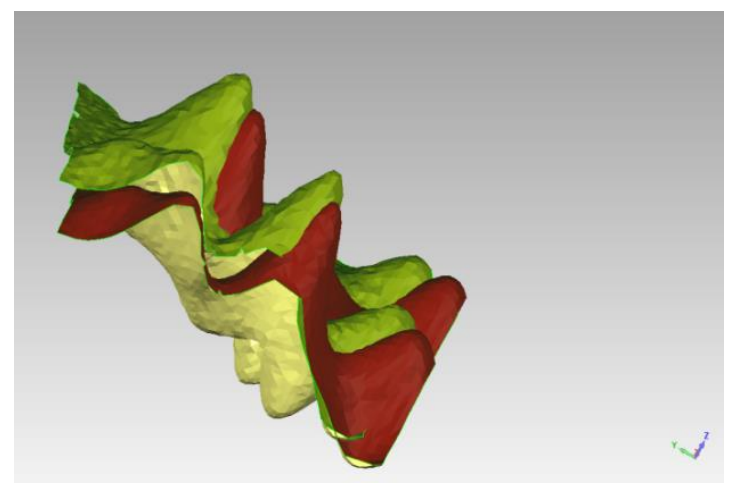

Figure 6. Template and search data.

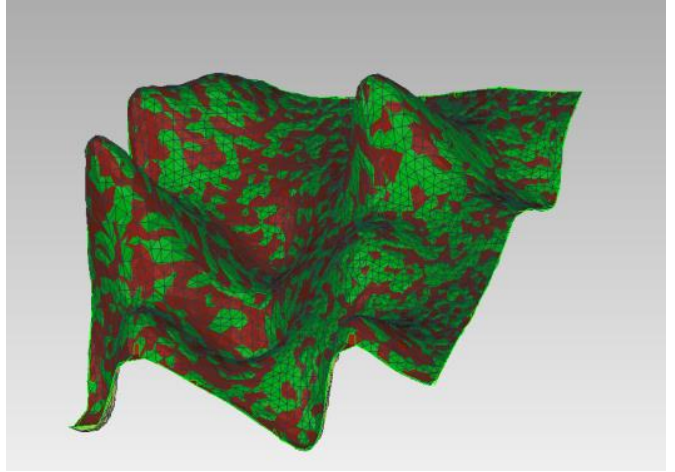

Figure 7. Template and transformed surface.

Table 4. Numerical results of 'wave' data

\begin{tabular}{lllclcccc}
\hline \hline Model & $\begin{array}{l}\text { No. of } \\
\text { Matched } \\
\text { Points }\end{array}$ & $\begin{array}{l}\sigma_{0} \\
(\mathrm{~mm})\end{array}$ & $\begin{array}{c}\sigma_{T_{x}} \\
(\mathrm{~mm})\end{array}$ & $\begin{array}{l}\sigma_{T_{y}} \\
(\mathrm{~mm})\end{array}$ & $\begin{array}{c}\sigma_{T_{z}} \\
(\mathrm{~mm})\end{array}$ & $\begin{array}{c}\sigma_{\omega} \\
(\mathrm{mm})\end{array}$ & $\begin{array}{c}\sigma_{\varphi} \\
(\mathrm{mm})\end{array}$ & $\begin{array}{c}\sigma_{\kappa} \\
(\mathrm{mm})\end{array}$ \\
\hline \multirow{2}{*}{ TLS_isotropic } & 3635 & 0.006377 & 0.000151 & 0.000151 & 0.000150 & 0.000101 & 0.000101 & 0.000076 \\
LS & 3907 & 0.008996 & 0.000145 & 0.000145 & 0.000144 & 0.000047 & 0.000047 & 0.000035 \\
TLS & 3634 & 5.014204 & 0.000326 & 0.000343 & 0.000152 & 0.000047 & 0.000034 & 0.000071 \\
\hline
\end{tabular}

\section{Conclusion and Discussion}

The motivation of this study is to investigate the error behaviours of parameter estimation of rigid-body transformation by applying EIV model which considers the both data sets are characterized as erroneous. The ommission of the stochastic properties of design matrix in the conventional Gauss-Markov model results in optimistic precision estimates. On the other hand, another assumption in almost all registration algorithms is the homogeneous and isotropic noise for both data sets. In fact this assumption is incorrect and unrealistic for 3-D data acquired by 3-D sensing such as stereovision and laser/ultrasonic range finders, because the accuracy is usually different between the depth direction and the direction orthogonal to it, resulting in an inhomogeneous and anisotropic noise distribution depending on the position, orientation, and type of the sensor [Kanatani]. Besides, data sets acquired with different sensors or data sets acquired in different times also have inhomogeneous and anisotropic noise level. In the case of a registration process (data fusion, change detection monitoring etc.) of this kind of data, the stochastic properties should be taken into consideration for an optimal, realistic solution.

By taking the stochastic properties of the elements of the design matrix into account, it is possible to have more realistic precision estimates of unknowns with the proposed model.

An implementation has been made in MATLAB computing language for the comparison of two mathematical models. We used anisotropic and non-homogeneous noise model as explained in stocahastic model chapter in our application for the template and search surfaces.

The experimental tests show that the proposed method provides the more realistic values but the magnitude of the difference is minor. The final sigma naught value is a statistically derived quantity and shows the amount of observation residuals. This value does not give any information about the accuracy, but we use sigma naught in $\sigma_{i}=\sigma_{0} \sqrt{q_{i i}}$ in order to calculate the a- 
posteriori sigma_i values of individual parameters. This situation indicates that the obtained a-posteriori covariance matrix in TLS3D is much smaller than the matrix in LS. On the other hand, results show that the TLS and LS methods do not differ in solution vector. In the view of these results, it is hard to state that TLS3D produces more correct results, but it would not be incorrect to say that it produces more reliable and realistic estimation values.

\section{References}

Akca, D., 2010. Co-registration of surfaces by 3D Least Squares matching. Photogrammetric Engineering and Remote Sensing, 76 (3), 307-318.

Akyilmaz, O.“ Total Least Squares Solution of Coordinate Transformation", Survey Review, 39, 303 pp.68-80 (January 2007)

Akyilmaz, O., 2011, Solution of the heteroscedastic datum transformation problems, Abstract of 1st Int. Workshop the Quality of Geodetic Observation and Monitoring Systems, April, 2011, Garching/Munich, Germany.

Besl, P., and McKay, H., "A method for registration of 3-D shapes," IEEE Transactions on pattern analysis and machine intelligence, vol. 14, 1992, p. 239-256.

Chen, Y.; Medioni, G.; , "Object modeling by registration of multiple range images," Robotics and Automation, 1991. Proceedings., 1991 IEEE International Conference on, vol., no., pp.2724-2729 vol.3, 9-11 Apr 1991

Gruen, A., 1985. Adaptive least squares correlation: A powerful image matching technique, S. Afr. Journal of Photogrammetry, Remote Sensing and Cartography, 14(3): 175-187.

Gruen, A., and D. Akca, 2005. Least squares 3D surface and curve matching, ISPRS Journal of Photogrammetry and Remote Sensing, 59(3):15-174.
Kanatani, K., Niitsuma, H., 2012,“Optimal Computation of 3-D Similarity:GaussNewton vs. Gauss-Helmert"Memoirs of the Faculty of Engineering, Okayama University, Vol. 46, pp. 21-33, January 2012

Kraus, K., C. Ressl, and A. Roncat, 2006. Least squares matching for airborne laser scanner data, Proceedings of the 5th International Symposium Turkish-German Joint Geodetic Days, 29-31 March, Berlin, Germany, unpaginated CD-ROM.

Maas, H.-G., 2002. Methods for measuring height and planimetry discrepancies in airborne laserscanner data, Photogrammetric Engineering \& Remote Sensing, 68(9):933-940.

Markovsky, I. S. Huffel, V., " Overview of total least squares methods ", Signal Processing 87 (2007) 2283-2302.

Neitzel, F., Petroviæ, S. ,2007, "Total Least Squares (TLS) im Kontext der Ausgleichung nach kleinsten Quadraten am Beispiel der ausgleichenden Geraden", ZFV, Vol. 133,141-148.

Ramos, J.A.; Verriest, E.I., 1997, "Total least squares fitting of two point sets in m-D," Decision and Control, 1997., Proceedings of the 36th IEEE Conference on , vol.5, no., pp.5048-5053 vol.5, 10-12 Dec 1997

Rusinkiewicz, S. and Levoy, M., 2001, "Efficient variants of the ICP algorithm," in Proc . 3rd Int. Conf. 3D Digital Imaging and Modeling (3DIM), Jun. 2001, pp. 145152.

Grant, D., Bethel, J., Crawford, M. (2012). Point-to-Plane Registration of Terrestrial Laser Scans, ISPRS Jpurnal of Photogrammetry and Remote Sensing 72, 16-12.

Salvi, J., Matabosch, C., Fofi, D., Forest, J. (2007). A Review of Recent Range Image Registration Methods wirh Accuracy Evaluation. Image and Vission Computing, Vol 25, no 2, pp 578-596. 
Lichti, D. D. (2007). Error Modelling, calibration and Analysis of an AM-CW Terrestrial Laser Scanner System, ISPRS Journal of Photogrammetry \& Remote Sensing, 61, 307-324.

Soudarissanane, S., Lindenbergh, R., Menenti, M., Teunissen, P. (2011). Scanning Geometry: Influencing Factor on the Quality of Terrestrial Laser Scanning Points, ISPRS Journal of Photogrammetry and Remote Sensing, 66, 389-399. 